Chapter 14

\title{
Cadmium Selenide-Gold (CdSe-Au) Hybrid Nanowires: Synthesis and Study of Charge Transfer
}

\author{
Xiaowei Teng and Xiaoxiong Jia \\ Additional information is available at the end of the chapter \\ http://dx.doi.org/10.5772/52828
}

\section{Introduction}

Complex hybrid nanostructures, which are assembled directly from different components without the aid from organic molecular bridges, are of major scientific and technological interest as they provide a variety of opportunities in various applications. By coupling components, each with its different functionalities, the hybrid nanostructures offer entirely novel properties that do not appear in their monocomponent counterparts. Many hybrid nanostructures, such as semiconductor/noble metal, semiconductor/semiconductor, noble metal/ noble metal, noble metal/magnetic metal or metal oxide, and semiconductor/magnetic metal or metal oxide, recently were synthesized via the Polyol processes, photochemical routes, seeded growth, or site-selective lithographic approaches. However, there is a limited ability to finely control the size, morphology, phase-segregation, and position of the components in hybrid nanostructures, partially reflecting the structural complexity imposed by the random nucleation of the second component.

Among various metals, gold $(\mathrm{Au})$ has been often studied in the hybrid system. The Au atom has the electron configuration of $(4 f)^{14}(5 d)^{10}(6 s)^{1}$. Due to s-p-d hybridization, a small amount of itinerant holes existing at the $\mathrm{d}$ band in bulk $\mathrm{Au}$ has been observed. The corresponding hybridization resulted in the electron configuration as of $(4 f)^{14}(5 d)^{10-x}(6 s)^{1+x}$. Thus, d-band holes can be generated in $\mathrm{Au}$, provide various interesting properties in magnetism, catalysis and photonics $[1,2,3]$.

Semiconductor nanomaterial have remarkable technological impacts on biomedical fluorescent labels, light emitting devices (LEDs), white-light laser sources, near-infrared emitters, solar cells, and tunable polarized lasers. Among various semiconductors, semiconductornoble metal hybrid structures are of particular interest for their great potential application in catalysis and photonics. The synthesis of II-VI and III-V semiconductors with precise 
controlling of various shapes has been intensively studied over the past decade. CdSe, as one of the II-VI semiconductors, becomes a popular choice for researchers due to its wellstudied synthetic approaches and promising applications in biological labeling and lightemitting diodes. CdSe nanostructures can be synthesized with various morphologies, such as spherical particle, rod, arrow, tear drop and tetrapod. Among these shapes, CdSe nanorods have been proved as good substrates for Au nanoparticles to grow on. Therefore, we selected CdSe nanorods as the host semiconductor to produce CdSe-Au hybrid nanorods in this work.

\section{Synthesis of CdSe Nanorod}

\subsection{Shape Control of CdSe}

The synthesis of II-VI and III-V semiconductors with precise controlling of various shapes has been intensively studied over the past decade. CdSe, as one of the II-VI semiconductors, becomes a popular choice for researchers due to its well-studied synthetic approaches and promising applications in biological labeling and light-emitting diodes. CdSe nanostructures can be synthesized with various morphologies, such as spherical particle, rod, arrow, tear drop and tetrapod. CdSe nanocrystals can be made via the injection of reactant precursors into a hot excess surfactant solvent, such as mixture of trioctylphosphine oxide (TOPO, $\left.\left[\mathrm{CH}_{3}\left(\mathrm{CH}_{2}\right)_{7}\right]_{3} \mathrm{PO}\right)$ and stearic acid $\left(\mathrm{CH}_{3}\left(\mathrm{CH}_{2}\right)_{16} \mathrm{COOH}\right)$, which usually provides a fast nucleation of CdSe seeds, followed by slow growth. Two injecting methods are mostly adopted. One is a mono-injection method, from which the spherical CdSe nanoparticles are formed. In this method, the temporal separation between nucleation and growth helps to achieve relatively narrow size distribution of nanoparticles. The other is a multiple-injection method, from which CdSe nanorods are produced.

In the early study of CdSe synthetic methods, Murray et al. provided a simple route to the production of nearly monodispersed $\mathrm{CdE}(\mathrm{E}=\mathrm{S}, \mathrm{Se}, \mathrm{Te})$ semiconductor nanoparticles [4]. A pre-heated $\left(\sim 300^{\circ} \mathrm{C}\right)$ surfactant solvent was prepared first followed by co-injection of organometallic precursors (cadmium dimethyl, $\mathrm{Cd}\left(\mathrm{CH}_{3}\right)_{2}$ and Se trioctylphosphine, TOP, $\left.\left[\mathrm{CH}_{3}\left(\mathrm{CH}_{2}\right)_{7}\right]_{3} \mathrm{P}\right)$ to produce a homogeneous nucleation using TOPO as a surfactant. The consequent growth at a moderate temperatures range $\left(230 \sim 260^{\circ} \mathrm{C}\right)$ resulted in the formation of uniform CdSe nanoparticles with diameters ranging from 1.5 to $11.5 \mathrm{~nm}$.

Peng et al. also developed Murray's method and first achieved the shape control of CdSe nanostructures from CdSe nanoparticles to CdSe nanorods using $\mathrm{Cd}\left(\mathrm{CH}_{3}\right)_{2}$ as precursor [5]. However, instead of using pure TOPO, the mixture of hexylphosphonic acid (HPA, $\left.\mathrm{CH}_{3}\left(\mathrm{CH}_{2}\right)_{5} \mathrm{P}(\mathrm{O})(\mathrm{OH})_{2}\right)$ and TOPO was used to serve as surfactant and reaction solvent. The function of HPA was to maintain the growth of CdSe nanorods in a controllable manner. Peng et al. also introduced multiple injections of organometallic precursors in the synthesis, which replenished monomers during the growth period of CdSe and resulted in the anisotropic growth of CdSe nanorods. By adopting HPA as surfactant and using multiple-injection method, CdSe nanorods were successfully produced in their studies. It was indicated 
that the growth of hexagonal crystal structure (wurtzite) of CdSe was highly anisotropic. Some growth conditions favored crystallization along the $<001>$ axis, usually called the c-axis of wurtzite structure, and resulted in the formation of the CdSe nanorods. However, the initial injection temperature adopted in this method was in the range from $340{ }^{\circ} \mathrm{C}$ to $360{ }^{\circ} \mathrm{C}$. Also, this multiple-injection method is relatively complex to execute compared to the monoinjection method adopted in the synthesis of CdSe nanoparticles.

Shieh et al. further explored Peng's multiple-injection approach in the synthesis of CdSe nanorods using cadmium mono-oxide $(\mathrm{CdO})$ as precursor, and n-tetradecylphosphonic acid (TDPA, $\mathrm{CH}_{3}\left(\mathrm{CH}_{2}\right)_{13} \mathrm{P}(\mathrm{O})(\mathrm{OH})_{2}$ ) and TOPO as surfactant [6]. They adopted a lower injection temperature $\left(260^{\circ} \mathrm{C}\right)$. However, they had to preheat the Se precursors to $120^{\circ} \mathrm{C}$ every time before the injection.

From above discussion we can extract three important factors in the synthesis of CdSe nanocrystals:

1. In the synthesis of CdSe nanoparticles, $\mathrm{Cd}(\mathrm{Ac})_{2}$ can be used as a precursor, avoiding high risk $\mathrm{Cd}\left(\mathrm{CH}_{3}\right)_{2}$;

2. a simple mono-injection method could be carried out using HPA as surfactant, avoiding the traditional co-injection method;

3. in the synthesis of nanorods, multiple-injection method is commonly used to synthesize nanorods.

In this work, we developed a mono-injection method based on traditional approach used in the synthesis of CdSe nanocrystals. We successfully achieved the shape control of CdSe nanostructures from nanoparticles to nanorods by simply adjusting the molar ratios between Se and Cd precursors. The growth models were proposed for different morphologies of CdSe and verified by the followed experimental results. The synthesized CdSe nanorods are utilized for the growth of Au nanoparticles which will be interpreted in details in the next section.

\subsection{Synthesis of CdSe}

In this work, we used $\mathrm{Cd}(\mathrm{Ac})_{2}$ as the $\mathrm{Cd}$ precursor, a mixture of TOPO, octadecylamine (ODA, $\mathrm{CH}_{3}\left(\mathrm{CH}_{2}\right)_{17} \mathrm{NH}_{2}$ ) and stearic acid as reaction solvent and surfactants. Se powder was dissolved in TOP to serve as injection solution for the growth of CdSe nanocrystals. The injection temperature was $310{ }^{\circ} \mathrm{C}$ and the growth time period for CdSe nanocrystals was 25 min. In a typical synthesis of CdSe, a mixture of $\mathrm{Cd}(\mathrm{Ac})_{2}(90 \%$, Strem Chemicals, $0.1132 \mathrm{~g}$ or $0.15 \mathrm{mmol})$, stearic acid (98\%, Alfa Aesar, $0.0853 \mathrm{~g}$ or $0.3 \mathrm{mmol})$, TOPO (98\%, Alfa Aesar, 2.5 g or $6.3 \mathrm{mmol}$ ) and ODA (90\%, Acros, $2.5 \mathrm{~g}$ or $8.3 \mathrm{mmol}$ ) were added into a $50 \mathrm{ml}$ three-neck round-bottom flask and then heated up via a heating mantle under vigorous stirring and argon flow protection. During the heating process, the initial white solid mixture turned into a transparent colorless solution. When the solution temperature reached $310{ }^{\circ} \mathrm{C}$, a Se solution made by dissolving $0.18,0.25,0.75$ or $1.0 \mathrm{mmol}$ Se powder ( $99 \%$, Strem Chemicals) into 1.79 , $2.02,5.38$ or $7.17 \mathrm{mmol}$ TOP $(90 \%$, Alfa Aesar) was quickly injected into the flask $(\ll<1 \mathrm{~s})$. A 
dark purple solution formed immediately. After heating for an additional $25 \mathrm{~min}$, the reaction was stopped by the removal of the heating mantle. After the solution was cooled to around $60{ }^{\circ} \mathrm{C}$, the reaction solution was equally distributed into two $14 \mathrm{ml}$ plastic centrifugation tubes. The final precipitation of CdSe was collected by washing it with ethanol and chloroform via a high speed centrifugation process.

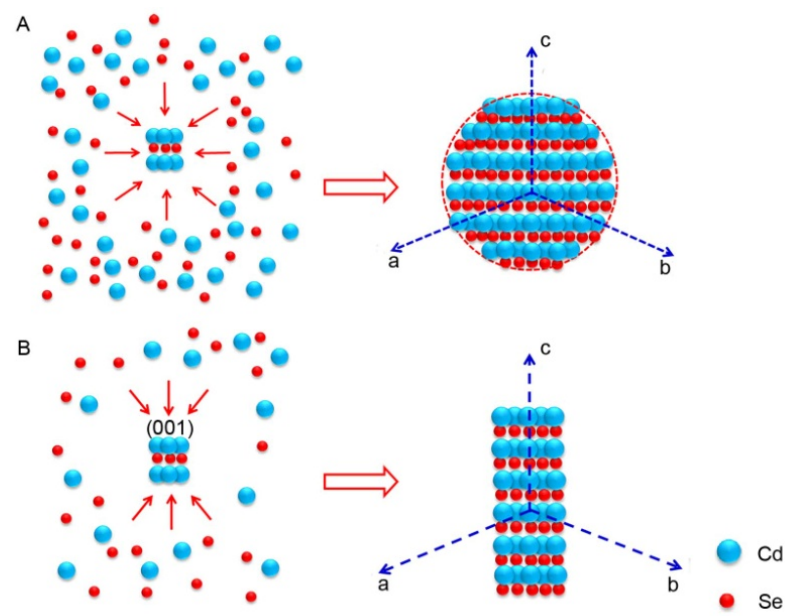

Figure 1. (A) Isotropic growth of CdSe nanoparticles. (B) Anisotropic growth of CdSe nanorods. (001) is the crystal surface perpendicular to the c-axis in the CdSe structure.

During the synthesis of $\mathrm{CdSe}, \mathrm{Cd}(\mathrm{Ac})_{2}$ first decomposed to form $\mathrm{Cd}$ atoms at high temperatures. These $\mathrm{Cd}$ atoms or clusters (aggregation of a few atoms) didn't nucleate before reaching the supersaturation. The formation of CdSe nanocrystals was triggered by injecting the Se/TOP solution into the flask. After injection, the Cd monomers reacted with Se to form CdSe monomers immediately, judging by the fast color changes upon the injection of Se into reaction solution. Once the concentration of CdSe reached supersaturation level, the CdSe nuclei (seeds) formed in the solution. In the meantime, CdSe monomers continued to deposit on CdSe nuclei, and resulted in the further growth of CdSe nanostructures. The continuous growth of CdSe nanostructures depleted the CdSe monomers in the solution, which would eventually decrease to the equilibrium concentration (the lowest concentration of CdSe monomer in solution). In the synthesis of CdSe with the molar ratio of $\mathrm{Se} / \mathrm{Cd}=5$, both nanorods and nanoparticles were observed. It is known that the formation of CdSe nanorods could be attributed to the elongation of c-axis in CdSe crystal structure. Namely, if the c-axis has a faster growth rate comparing to other axes in CdSe crystal structure, CdSe nanorods will be formed via an anisotropic growth along c-axis; if all the axes have a similar growth rate, CdSe nanoparticles will form via an isotropic growth. The growth rates of different axes can be also affected by the concentration of monomers in the solution. If the concentration of monomers is very high, all the axes will have a similar growth rate, CdSe 
nanoparticles will be formed; if the concentration of the monomers is low, CdSe nanorods will be formed via a dominant growth along c-axis. The corresponding scheme for the isotropic and anisotropic growth of CdSe nanocrystals is shown in Figure 1.

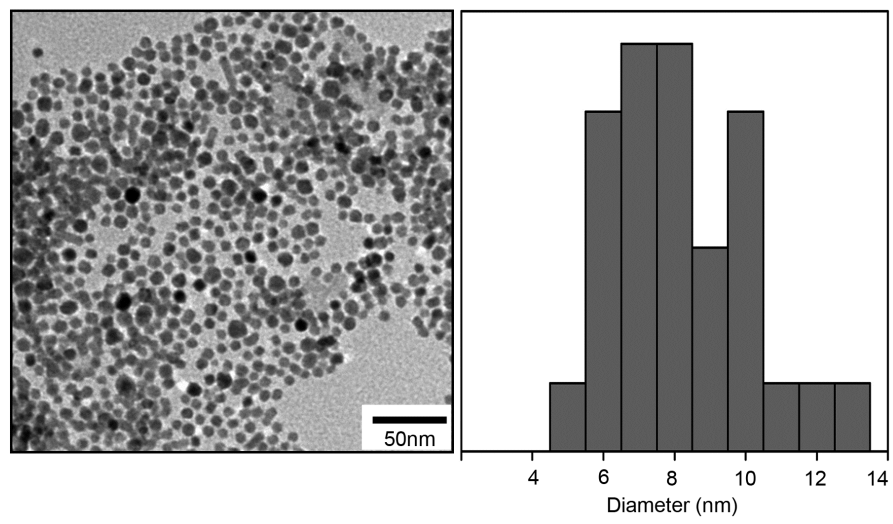

Figure 2. TEM images of $\mathrm{CdSe}$ nanoparticles and the corresponding sizing histogram. These CdSe nanoparticles were synthesized at $310^{\circ} \mathrm{C}$ for $25 \mathrm{~min}$ using $\mathrm{Cd}(\mathrm{Ac})_{2}(0.15 \mathrm{mmol}), \mathrm{CH}_{3}\left(\mathrm{CH}_{2}\right)_{16} \mathrm{COOH}(0.3 \mathrm{mmol}), \mathrm{TOPO}(2.5 \mathrm{~g}), \mathrm{ODA}(2.5 \mathrm{~g}), \mathrm{Se}$ $(1 \mathrm{mmol})$ and TOP $(7.17 \mathrm{mmol})$. The molar ratio of Se/Cd was 6.7. The average diameter of CdSe nanoparticles was 7.7 $\pm 1.9 \mathrm{~nm}$.

In order to verify the effect of CdSe concentration on the morphologies of CdSe as proposed in Figure 1, a series of experiments was conducted using different Se/Cd molar ratios. To simplify the experimental procedure, we kept the amount of $\mathrm{Cd}(\mathrm{Ac})_{2}$ constant, and only adjusted the amount of Se to change the molar ratios of Se/Cd in the syntheses. If the model we proposed is valid, high amount of Se will lead to the formation of CdSe nanoparticles via the isotropic growth; while fewer amount of Se will lead to the formation of CdSe nanorods by encouraging the growth along c-axis. The TEM images of resulting CdSe nanostructures are shown in Figure 2 and 3. When Se/Cd molar ratio was equal to 6.7, CdSe crystals grow isotropically during the reaction due to the abundant existence of monomers in the solution (Figure 2). When the Se/Cd molar ratios decreased to 1.7 and 1.2 in the syntheses, the CdSe nanorod systems were synthesized (Figures 3). The observations are well consistent with the growth models we proposed. Moreover, the experimental procedure presenting in this work is more easily to conduct. This method allows the synthesis to conduct at a moderate temperature. Also by simply changing the molar ratios between Se and Cd, different shaped CdSe nanocrystals can be successfully synthesized, which provides a new thought to control the shape of CdSe nanocrystals.

In summary, a simple mono-injection method was adopted in the synthesis of CdSe nanocrystals. CdSe nanoparticles and nanorods were produced by adjusting the molar ratios between Se and Cd precursors. We proposed a plausible growth model in which the concentration of monomers in the solution played an important role in determining the final morphologies of CdSe nanocrystals. The model can be briefly described as high concentra- 
tions of CdSe monomer lead to an isotropic growth of CdSe nanoparticles; while low concentrations of CdSe monomer lead to an anisotropic growth of CdSe nanorods. A series of experiments using different $\mathrm{Se} / \mathrm{Cd}$ molar ratios $(\mathrm{Se} / \mathrm{Cd}=6.7,1.7$ and 1.2$)$ was carried out to verify this growth model.

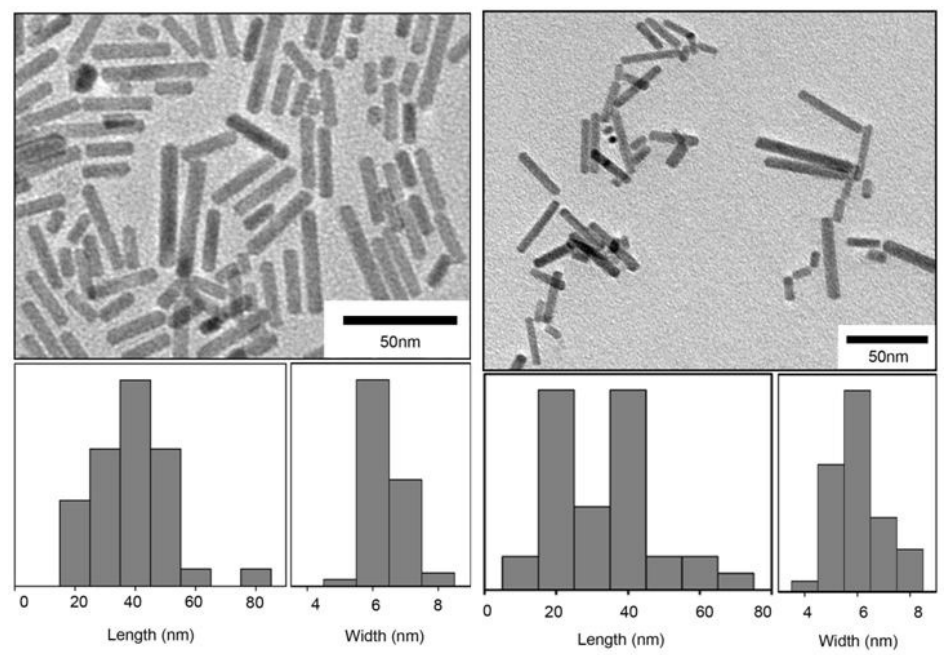

Figure 3. TEM images of CdSe nanorods and the corresponding sizing histograms. (Left) These CdSe nanorods were synthesized at $310^{\circ} \mathrm{C}$ for $25 \mathrm{~min}$ using $\mathrm{Cd}(\mathrm{Ac})_{2}(0.15 \mathrm{mmol}), \mathrm{CH}_{3}\left(\mathrm{CH}_{2}\right)_{16} \mathrm{COOH}(0.3 \mathrm{mmol}), \mathrm{TOPO}(2.5 \mathrm{~g}), \mathrm{ODA}(2.5 \mathrm{~g}), \mathrm{Se}$ $(0.25 \mathrm{mmol})$ and TOP $(2.02 \mathrm{mmol})$. The molar ratio of $\mathrm{Se} / \mathrm{Cd}$ was 1.7 . The length of the rod was $33.4 \pm 13.0 \mathrm{~nm}$, the width was $5.9 \pm 0.5 \mathrm{~nm}$. (Right) These CdSe nanorods were synthesized at $310{ }^{\circ} \mathrm{C}$ for $25 \mathrm{~min}$ using $\mathrm{Cd}(\mathrm{Ac})_{2}(0.15$ $\mathrm{mmol}), \mathrm{CH}_{3}\left(\mathrm{CH}_{2}\right){ }_{16} \mathrm{COOH}(0.3 \mathrm{mmol})$, TOPO $(2.5 \mathrm{~g})$, ODA $(2.5 \mathrm{~g})$, Se $(0.15 \mathrm{mmol})$ and TOP $(1.79 \mathrm{mmol})$. The molar ratio of $\mathrm{Se} / \mathrm{Cd}$ was 1.2. The length of the rod was $27.0 \pm 14.4 \mathrm{~nm}$, the width was $5.6 \pm 0.9 \mathrm{~nm}$.

\section{Synthesis of CdSe-Au Nanorod}

\subsection{Material Synthesis}

The growth of metals on semiconductors as a means of increasing functionalities is an important subject in material science. A preparation of such hybrids usually involves the injection of a metal salt into the semiconductor nanocrystal solution. Mokari et al. first achieved a position-selective growth of $\mathrm{Au}$ nanoparticles onto CdSe nanorods by gradually adding gold chloride $\left(\mathrm{AuCl}_{3}\right)$ solution into $\mathrm{CdSe}$ nanorods solution at room temperature in the presence of dodecyldimethylammonium bromide (DDAB, $\left[\mathrm{CH}_{3}\left(\mathrm{CH}_{2}\right)_{11}\right]_{2} \mathrm{~N}\left(\mathrm{CH}_{3}\right)_{2} \mathrm{Br}$,) and dodecylamine $\left(\mathrm{CH}_{3}\left(\mathrm{CH}_{2}\right)_{11} \mathrm{NH}_{2}\right)$ [7]. They claimed that amine reduced $\mathrm{AuCl}_{3}$ to form $\mathrm{CdSe} / \mathrm{Au}$ hybrid with Au nanoparticles deposited on the tips of CdSe nanorods. Furthermore, by controlling the reaction times, the sizes of Au nanoparticles could be tuned from 2 to $4 \mathrm{~nm}$. The most compelling aspect of Mokari's work was the high selective growth onto the rod's tips. 
They attributed this position-selective growth to higher surface energies of the tips, and more accessible sites due to imperfect passivation of surfactants on the tips of CdSe nanorods. In their follow-up work, Mokari et al. further studied the growth process of Au nanoparticles on CdSe nanorods [8]. At long reaction times, the concentration of $\mathrm{AuCl}_{3}$ in solution dropped to a critical value and the reaction transitioned from a growth state to a ripening state, resulting in the disappearance of small Au nanoparticles along the surface of nanorod and the growth of large Au nanoparticles on the tips of nanorod. From the above discussion we can conclude two important factors to achieve the growth of Au nanoparticles on CdSe nanorods:

1. the growth of Au nanoparticles onto CdSe nanorods can be realized via a room-temperature reaction in the presence of DDAB and dodecylamine;

2. longer reaction time yields larger Au nanoparticles due to the ripening process.

Since in the ideal Au-based DMS, the growth of small Au nanoparticles has to achieve a uniform distribution all over the surfaces of CdSe nanorods, not only on some particular sites on CdSe nanorods, an alternative method to achieve the uniform growth of Au nanoparticles all over the surfaces of CdSe nanorods is required.

The Au precursor stock solution was prepared by dissolving $12 \mathrm{mg}(0.04 \mathrm{mmol})$ of $\mathrm{AuCl}_{3}$ (99.99\%, Alfa Aesar), $15 \mathrm{mg}$ (0.05 mmol) of 1-dodecyltrimethylammonium bromide (DTAB, $\mathrm{C}_{12} \mathrm{H}_{25}\left(\mathrm{CH}_{3}\right)_{3} \mathrm{NBr}, 99 \%$, Alfa Aesar) in $2 \mathrm{ml}$ of chloroform at room temperature. The color of the $\mathrm{AuCl}_{3}$ solution changed to yellow from orange after 5 min sonication. $0.15 \mathrm{mmol}$ of assynthesized CdSe nanorods with $30 \mathrm{mg}$ TOPO $\left(\mathrm{C}_{24} \mathrm{H}_{51} \mathrm{OP}, 98 \%\right.$, Alfa Aesar $)$ and $30 \mathrm{mg}$ Octadecylamine (ODA, $\mathrm{CH}_{3}\left(\mathrm{CH}_{2}\right)_{17} \mathrm{NH}_{2}, 90 \%$, Acros) were dissolved in $12 \mathrm{ml}$ of chloroform in a $50 \mathrm{ml}$ three-neck round-bottom flask under vigorous stirring and argon protection. Au precursor stock solution $(0.167 \mathrm{ml})$ containing $1 \mathrm{mg}(0.003 \mathrm{mmol})$ of $\mathrm{AuCl}_{3}$ was injected into the reaction flask within 10 seconds. The reaction was carried out under room temperature. The TEM samples of CdSe-Au hybrid nanorods were prepared by taking $0.3 \mathrm{ml}$ reaction solution from the flask at several time intervals after the initial injection. After 5, 7, 10, 30 or 60 min, the samples were collected into a $14 \mathrm{ml}$ centrifuge tube and centrifuged for $5 \mathrm{~min}$ to get the final products.

The galvanic replacement reaction usually involves a redox process. The driving force of this process is the reduction potential $\left(\mathrm{E}^{\circ}\right)$ difference between two reactants, with one reactant acting as the oxidizer and the other reactant as the reducer. Since $\mathrm{E}^{\circ}\left(\mathrm{Au}^{3+} / \mathrm{Au}^{0}\right)$ has higher reduction potential than $\mathrm{E}^{\circ}\left(\mathrm{Se}^{2-} / \mathrm{Se}^{4+}\right)$, the following reaction would happen in the solution:

$$
2 \mathrm{AuCl}_{3}+\mathrm{CdSe} \rightarrow 2 \mathrm{Au}+\mathrm{Cd}^{2+}+\mathrm{Se}^{4+}+6 \mathrm{Cl}^{-}
$$

After mixing with CdSe, $\mathrm{Au}^{3+}$ ions were reduced by $\mathrm{Se}^{2-}$ to form $\mathrm{Au}$ atoms at the surfaces of CdSe nanorods. As reaction went on, Au nuclei formed on the surfaces of CdSe nanorods, as shown in Figure 4. 

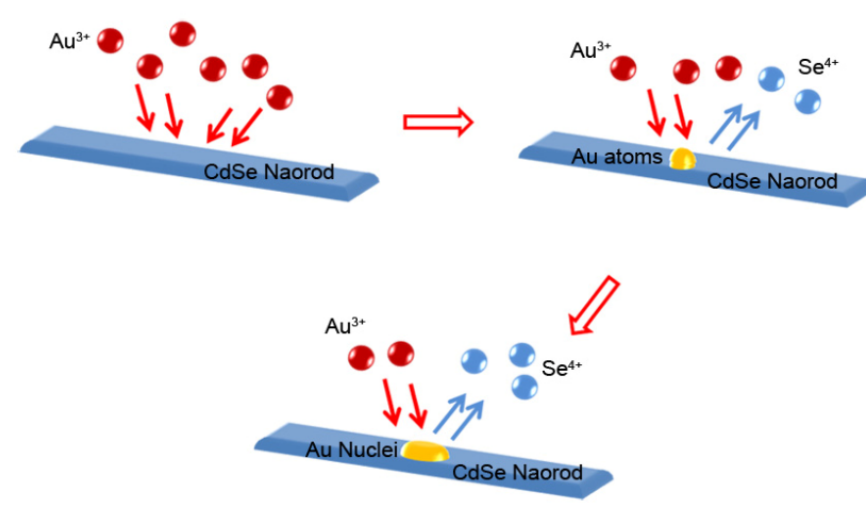

Figure 4. The galvanic replacement reactions between $\mathrm{CdSe}$ and $\mathrm{AuCl}_{3}$ and the formation of the $\mathrm{Au}$ nuclei.

\subsection{Time Effect}

In the syntheses of CdSe-Au hybrid nanorods, the reaction times varied from 5 to 60 min to control the sizes of Au nanoparticles (See selected TEM images of CdSe-Au synthesized at 5, 10, and 30 mins in Figure 5). The CdSe nanorods used for reaction were synthesized using a $\mathrm{Se} / \mathrm{Cd}$ molar ratio of 1.7. The molar numbers of CdSe nanorods and $\mathrm{AuCl}_{3}$ was $0.15 \mathrm{mmol}$ and $0.003 \mathrm{mmol}$. The growth of $\mathrm{Au}$ nanoparticle on CdSe nanorods with respect to reaction time was shown in Figure 6. From the curve we can see that in the first $10 \mathrm{~min}$, the average size of $\mathrm{Au}$ nanoparticles increased sharply from 0 to $2.3 \pm 0.7 \mathrm{~nm}$; after 10 min reaction, $\mathrm{Au}$ nanoparticles exhibited a rather stagnant growth: sizes changed from $2.3 \pm 0.7 \mathrm{~nm}$ at $10 \mathrm{~min}$ to $2.4 \pm 0.9 \mathrm{~nm}$ at $60 \mathrm{~min}$. The time-dependent growth of Au nanoparticles can be attributed to the change of concentration of $\mathrm{AuCl}_{3}$ in the solution at different reaction times. The formation of $\mathrm{Au}$ nanoparticles was triggered by the injection of $\mathrm{AuCl}_{3}$. At the initial time point $(t=0)$, there was no $\mathrm{Au}$ nanoparticle observed. Once the $\mathrm{AuCl}_{3}$ was injected into the solution, the reaction between $\mathrm{Au}^{3+}$ and $\mathrm{Se}^{2-}$ occurred subsequently at the surfaces of the CdSe nanorods. This reaction led to the formation of Au atoms on the surfaces of CdSe nanorods. In the first $10 \mathrm{~min}$, the Au nanoparticles kept growing at a relatively rapid growth rate due to the abundant $\mathrm{AuCl}_{3}$ in the solution. As more Au nanoparticles formed in the solution, the concentration of $\mathrm{Au}^{3+}$ decreased until reaching the equilibrium. The growth rate of $\mathrm{Au}$ nanoparticles slowed down and ultimately suppressed by the depletion of the $\mathrm{Au}^{3+}$, and the solution was considered to approach the equilibrium state. At an equilibrium state, the growth of Au nanoparticles transitioned to ripening state. 

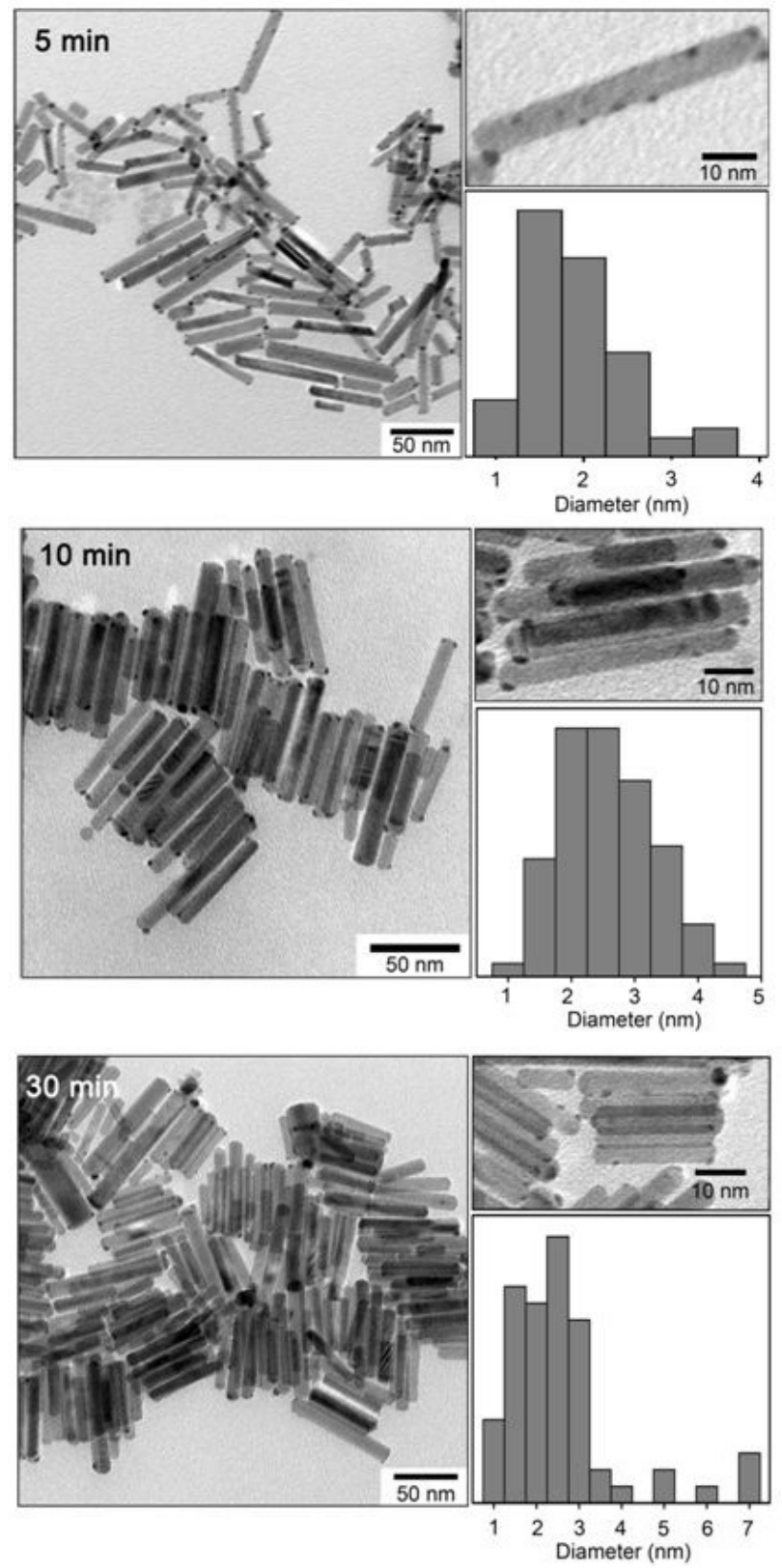

Figure 5. Original and enlarged TEM images of CdSe-Au hybrid nanorods and Au sizing histogram after 5, 10 and 30 min reactions. The average diameters of Au nanoparticles were $1.9 \pm 0.8 \mathrm{~nm}, 2.3 \pm 0.7 \mathrm{~nm}$ and $2.3 \pm 1.3 \mathrm{~nm}$, respectively. 


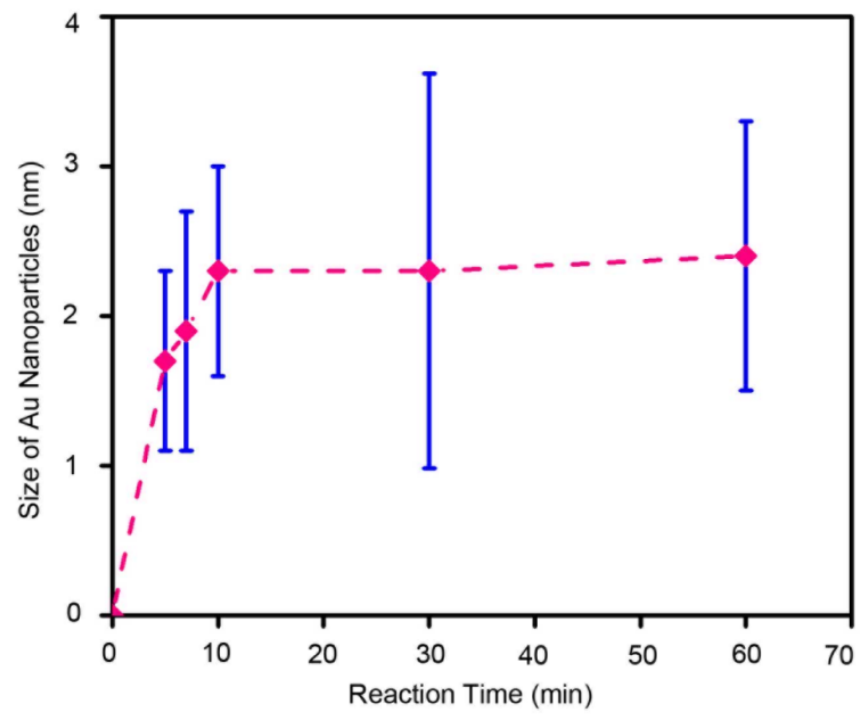

Figure 6. Changes of Au nanoparticles sizes as a function of reaction time

\subsection{The Ostwald Ripening Process in CdSe-Au Solution}

The Ostwald ripening process is the growth of large nanoparticles at the expense of dissolution of small ones at an equilibrium state. It is a spontaneous process because large nanoparticles are more thermodynamically stable than small ones. After the solution reached equilibrium state probably after 10 minutes reactions, large Au nanoparticles kept growing while small ones disappeared. Mokari1 et al. first proposed a growth mechanism for this process that small Au nanoparticles first released electrons into solution and then transferred these electrons to large Au nanoparticles through CdSe nanorods [8]. After giving out electrons, the small Au nanoparticles escaped from the surfaces of nanorods and became free $\mathrm{Au}$ ions in the solution. These $\mathrm{Au}$ ions re-deposited on large $\mathrm{Au}$ nanoparticles and were reduced to $\mathrm{Au}^{0}$ (Figure 7.). The result of this process was disappearance of small $\mathrm{Au}$ nanoparticles and growth of large ones. However, they did not provide direct evidence to prove that small $\mathrm{Au}$ particles can release the electrons automatically. Also, no observation of electric current in CdSe nanorod was reported. Here, we proposed an alternative growth mechanism in which the growth of the Au nanoparticles is attributed to the Ostwald ripening process.

From a standpoint of kinetics, when the concentration of $\mathrm{AuCl}_{3}$ was high, formation of small $\mathrm{Au}$ nanoparticles was dominant. When $\mathrm{Au}$ atoms/clusters monomers were depleted, the whole system transited from a fast growth state (kinetics state) to a ripening state (thermo- 
dynamics state). At the ripening state, from a standpoint of thermodynamics, small $\mathrm{Au}$ nanoparticles on the surfaces of CdSe nanorods dissolved into solution in the form of $\mathrm{Au}$ atoms (monomers) and re-deposited on the surfaces of large Au nanoparticles. As a result of this ripening process, the small Au nanoparticles dissolved to favor the growth of large particles on the CdSe nanorods. Figure 8 shows the Ostwald ripening process in CdSe-Au hybrid nanorods solution at equilibrium state. As the reaction continued, we found that $\mathrm{Au}$ nanoparticles tend to migrate to the tips of CdSe nanorods. After 5 minutes reactions, (Figure 6), small Au nanoparticles were found all over the surfaces of CdSe nanorods. After 30 and 60 minutes reactions, Au nanoparticles were found mostly at the tips of CdSe nanorods. This migration of Au nanoparticles can be attributed to higher surface energies on the tips of nanorod, resulting from more accessible sites on the tips where surfactant capping was weak. Therefore, the tips of nanorods are preferential growing sites for Au nanoparticles at long reaction times.

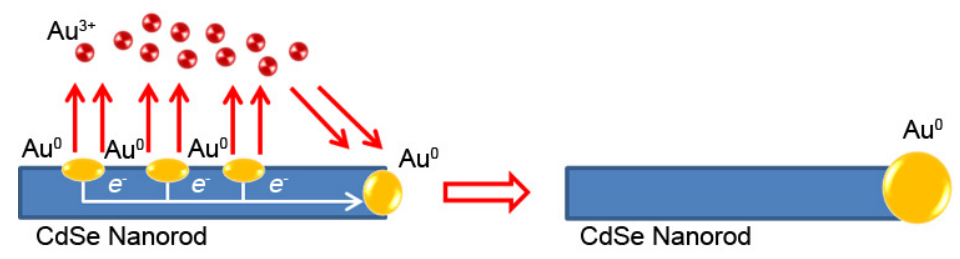

Figure 7. The growth process proposed by Mokari1 et al. Small Au nanoparticles escape to the solution as free Au ions by release their electrons. The released electrons transfer to large Au nanoparticles to reduce Au ions in the solution and results in the growth of large Au nanoparticles.
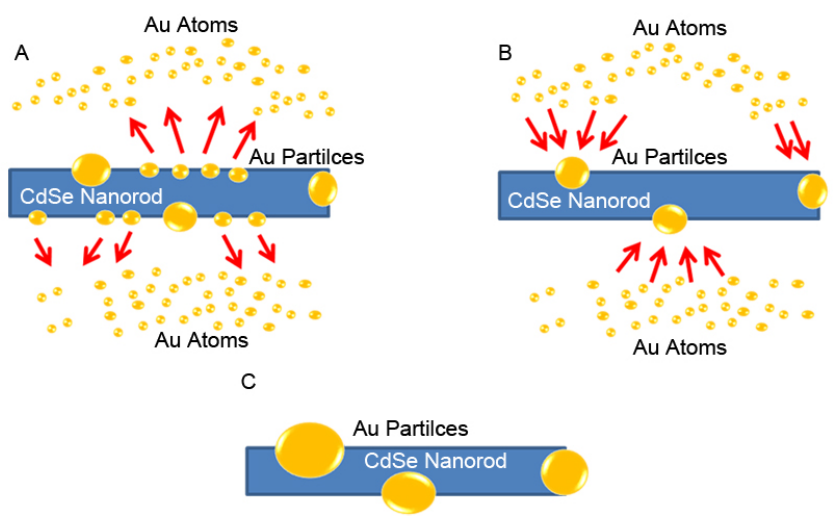

Figure 8. The Ostwald ripening process in CdSe-Au solution. (A) dissolution of small Au particles; (B) growth of large Au particles; (C) CdSe-Au hybrid nanorods with large Au particles. 


\subsection{In-Situ Growth of Au Nanoparticles under Electron Irradiation in TEM}

A unique growth of $\mathrm{Au}$ nanoparticles was observed under the irradiation of the electron beams in TEM. Figure 9 shows this process. Figure 9A is the original image of CdSe-Au nanorods after 7 min reactions exposed under electron beam of low intensity, Figure $9 \mathrm{~B}$ is the same sample exposed under electron beam of high intensity for $1 \mathrm{~min}$. From Figure 9A to $\mathrm{B}$ we can see that as the electron beam intensity increased, the average size of Au nanoparticles experienced a clear growth from the initial $2.3 \pm 0.7 \mathrm{~nm}$ to the final $5.0 \pm 1.8 \mathrm{~nm}$. This unique growth of $\mathrm{Au}$ nanoparticles under electron irradiation can be attributed to the size effect on the melting point of Au nanoparticles. It has been approved that the melting points of nanoparticles have great dependencies to their sizes. Typically, particles exhibit decreasing melting points with decreasing sizes. This size-dependent melting point of nanoparticles can be calculated through the Gibbs-Thomson equation:

$$
T_{M}(d)=T_{M B}\left(1-\frac{4 \sigma_{s l}}{\rho_{s} d H_{f}}\right)
$$

where $T_{M B}$ is bulk melting temperature, $\sigma_{s l}$ is solid liquid interface energy, $H_{f}$ is bulk heat of fusion, $\rho_{s}$ is density of solids and $d$ is particle diameter. From this equation we can see that as particle diameter $d$ increases, the particle melting temperature $T_{M}(d)$ decreases. The melting point of bulk Au metal is $1336 \mathrm{k}\left(1063^{\circ} \mathrm{C}\right)$. If the size of Au nanoparticle is $1.6 \mathrm{~nm}$ (the smallest $\mathrm{Au}$ nanoparticles we observed), the corresponding melting point is only $554 \mathrm{~K}$ (281 ${ }^{\circ} \mathrm{C}$ ) (The parameters for calculation are in Table 1). Also, the operation of TEM is under vacuum, which further decreases the melting points of small Au nanoparticles. When Au nanoparticles were exposed to the electron beam in TEM, small Au nanoparticles melted easily due to their lower melting points. In the meantime, these melted Au particles were migrating towards large Au nanoparticles in order to achieve lower surface energies thermodynamically. The result of the process was the melting of small particles and the growth of large ones. The observed phenomenon also indicated that small Au nanoparticles distributed all over the surface of nanorod in the original sample. Otherwise, there were not enough growth resources for this dramatic growth of Au nanoparticles.

\begin{tabular}{ccccccc}
\hline$d / \mathrm{m}$ & $\sigma_{s l} / \mathrm{J} \mathrm{m}^{-2}$ & $H_{f} / \mathrm{J} \mathrm{Kg}^{-1}$ & $\rho_{s} / \mathrm{Kg} \mathrm{m}^{-3}$ & $T_{M B} / \mathrm{K}$ & $T_{M}(d) / \mathrm{K}$ & $T_{M}(d) /{ }^{\circ} \mathrm{C}$ \\
\hline $1.6 * 10^{-9}$ & 0.27 & $6.27 * 10^{4}$ & $1.84^{*} 10^{4}$ & 1336 & 554 & 281
\end{tabular}

Table 1. Parameters for Calculating the Melting Point of Au Nanoparticle 

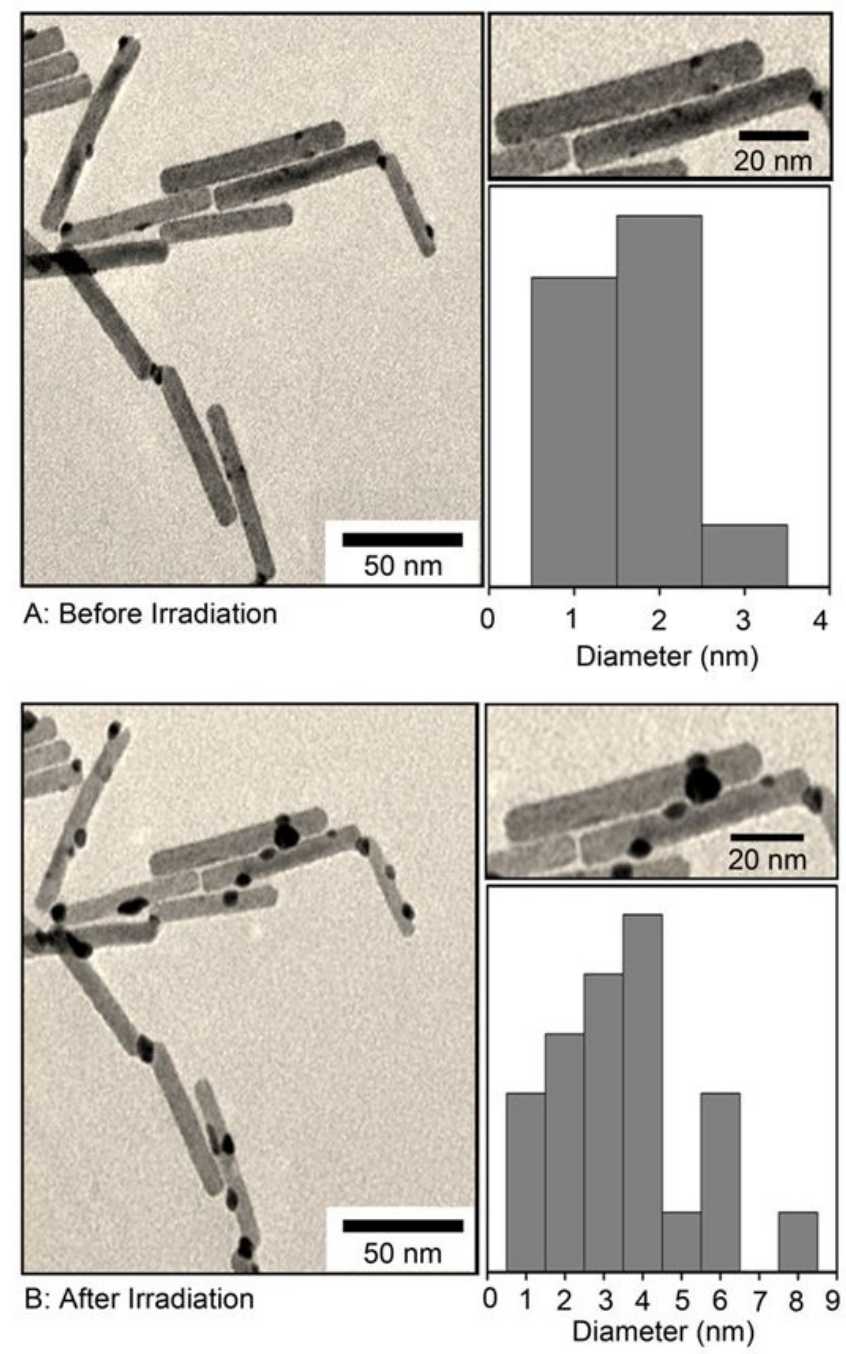

Figure 9. TEM images of CdSe-Au synthesized using $0.003 \mathrm{mmol} \mathrm{AuCl}_{3}$ into $0.15 \mathrm{mmol} \mathrm{CdSe}$ nanorods: (A) before irradiation (B) after irradiation. The diameters of Au nanoparticles were (A) $2.3 \pm 0.7 \mathrm{~nm}$ and (B) $5.0 \pm 1.8 \mathrm{~nm}$.

\section{Electronic Structure Characterized by X-ray Absorption Near Edge Spectroscopy (XANES)}

XANES is a powerful X-ray absorption spectroscopy technique for investigating charger transfer between distinctive elements as well as oxidation states of specific element within 
the nanomaterials [9]. By analyzing absorption signals in XANES, one can extract abundant chemical information of the studied material, like formal valence, coordination environment, the unoccupied band structure of a material, and fingerprint a mixture of sites/ compounds (e.g. oxidation state determination).

Since XANES is sensitive to the local electronic structure, it has been used to study the electronic redistribution or charge transfer upon alloying between various compounds. Obviously, it would also be the desirable technique to study the electronic structure of those $\mathrm{CdSe} / \mathrm{Au}$ hybrid nanowires. Despite the fact that $\mathrm{Au}$ metal with filled $\mathrm{d}^{10}$ orbital usually doesn't exhibit an intense white line (the first feature in the absorption edge) at $\mathrm{L}_{3}$ edge thresholds compared with unfilled $\mathrm{d}^{9}$ metals (e.g. Pt), the orbital $\mathrm{s}-\mathrm{p}-\mathrm{d}$ hybridization results in a $5 d^{10-x} 6(s p)^{1+x}$ electron configuration, leading to a potentially noticeable increase of white line intensity. The changes of the number of holes in the $\mathrm{d}$ band reflect charge transfer between various CdSe-Au hybrid nanostructures with different sizes of $\mathrm{Au}$ components. Therefore, enhancement of the white line intensity from $\mathrm{Au} \mathrm{L}_{3}$ edges can thus be used as a sensor, to detect and quantify the hybrid formation. Figure 10 shows normalized $\mathrm{Au}_{3}$ edge XANES spectra of CdSe-Au hybrids and that of Au foil for reference. It can be seen that the strength of the $\mathrm{Au}$ white line feature in $\mathrm{L}_{3}$ edge is noticeably lower than that of pure Au foil as the size of $\mathrm{Au}$ components decreased. Since white line features in $\mathrm{Au} \mathrm{L}_{3}$ edge arise from $2 p_{3 / 2}$ to $5 d_{5 / 2}$ dipole transitions, the observations indicate a decrease in the number of unoccupied states of $\mathrm{d}$ character at the Au site upon the decreasing the size of Au components. The data can be further interpreted in terms of gain of d charge in Au site. These findings dramatically highlight the intriguing structure-property relationship of nanoscaled electronic materials, and deserve further investigations.
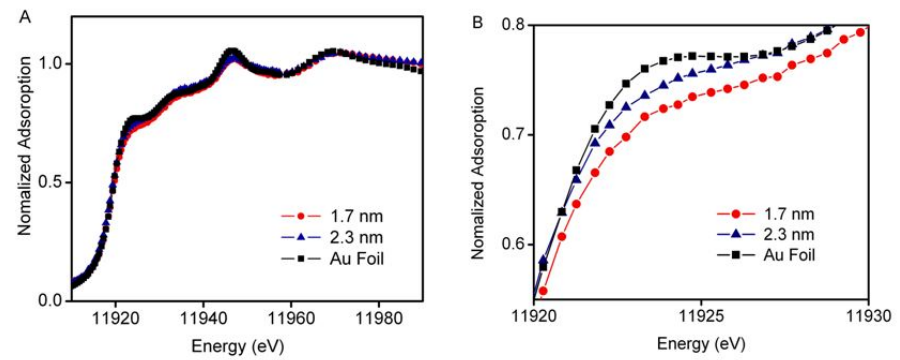

Figure 10. Normalized XANES spectra of Au in the CdSe-Au hybrid nanorods.

\section{Conclusion}

The target of this work was to synthesize CdSe-Au hybrid nanorods. The first step was to synthesize CdSe nanorods. A simple mono-injection method was adopted in the syntheses. 
By reducing the molar ratios between Se and Cd, we have successfully achieved the shape control of CdSe nanocrystals from nanoparticles to nanorods. We found 5 to1 was a critical molar ratio between Se and $\mathrm{Cd}$ to form different CdSe nanostructures. When the molar ratio between Se and Cd was higher than 5 (6.7), CdSe nanoparticles were synthesized. When the molar ratio was lower than 5 (1.2 and 1.7), CdSe nanorods were synthesized. The growth models for different CdSe nanostructures were proposed and validated by the subsequent experimental results. In the model, the concentration of monomer plays an important role in the shape control of CdSe nanostructures. High concentration of monomer leads to the isotropic growth of CdSe nanoparticles. Low concentration of monomer leads to the anisotropic growth of CdSe nanorods. Comparing with traditional approach adopted in the shape control of CdSe nanostructures, the synthesis approach used in this work is more easy to conduct; also a relatively moderate reaction temperature and more favorable $\mathrm{Cd}$ precursor make the synthesis approach safer.

The growth of $\mathrm{Au}$ nanoparticles on CdSe nanorods involves galvanic replacement reactions between $\mathrm{AuCl}_{3}$ and $\mathrm{CdSe}$ nanorods at room temperature. In the reaction, $\mathrm{Au}^{3+}$ was reduced to $\mathrm{Au}$ atoms while $\mathrm{Se}^{2-}$ was oxidized to $\mathrm{Se}^{4+}$. By adjusting the reaction times, Au nanoparticles with diameter from $1.7 \pm 0.6 \mathrm{~nm}$ to $2.4 \pm 0.9 \mathrm{~nm}$ could be synthesized on the surfaces of CdSe nanorods. Before the reaction closing to equilibrium state, Au nanoparticles exhibited a fast growth rate due to a high concentration of $\mathrm{AuCl}_{3}$ in solution. When the concentration of $\mathrm{AuCl}_{3}$ approaching to equilibrium level, the reaction was closely related to the Ostwald ripening process in which the small Au nanoparticles dissolved into solution and the mass was transferred to increase the size of the large nanoparticle on the nanorods.

\section{Acknowledgements}

This work is supported by the University of New Hampshire. Use of the National Synchrotron Light Source, Brookhaven National Laboratory, was supported by the U.S. Department of Energy, Office of Science, Office of Basic Energy Sciences, under Contract DEAC02-98CH10886 and beam lines X19A/X18B are partly supported by Synchrotron Catalysis Consortium under contract DE-FG02-05ER15688.

\section{Author details}

Xiaowei Teng ${ }^{*}$ and Xiaoxiong Jia

*Address all correspondence to: xw.teng@unh.edu

University of New Hampshire, USA 


\section{References}

[1] Saunders, A. E., Popov, I., \& Banin, U. (2006). Synthesis of Hybrid CdS-Au Colloidal Nanostructures,. J. Phys. Chem. B, 110, 25421-25429.

[2] Crespo, P., Litran, R., Multigner, M., de la Fuente, J. M., Sanchez, J. C., Lopez, M. A., Garcia, C., Lopez, Cartes. A., Hernando, S., \& Penades, A. Fernandez. (2004). Permanent magnetism, magnetic anisotropy, and hysteresis of thiol-capped gold nanoparticles,. Phys. ReV. Lett. , 93, 087204.

[3] Zhong, C. J., \& Maye, M. M. (2001). Core-shell assembled nanoparticles as catalysts. Advanced Materials, 13, 1507-1511.

[4] Murray, C. B., Noms, D. J., \& Bawendi, M. G. (1993). Synthesis and characterization of nearly monodispersed $\mathrm{CdE}(\mathrm{E}=\mathrm{S}, \mathrm{Se}, \mathrm{Te})$ semiconductor Nano crystallites. J. Am. Chem. Soc., 115, 8706-8715.

[5] Peng, X. G., Manna, L., Yang, W., Wickham, J., Escher, E., Kadavanich, A., \& Alivisatos, A. P. (2000). Shape control of CdSe nanocrystals. Nature, 404, 59-61.

[6] Shieh, F., Saunders, A. E., \& Korgel, B. A. (2005). General shape control of colloidal CdS, CdSe, CdTe quantum rods and quantum rod heterostructures,. Phys. Rev. B. Lett, 109, 8538-8542.

[7] Mokari, T., Rothenberg, E., Popov, I., Costi, R., \& Banin, U. (2004). Selective Growth of Metal Tips onto Semiconductor Quantum Rods and Tetrapods,. Science. , 304, 1787-1789.

[8] Mokari, T., Sztrum, C. G., Salant, A., Rabani, E., \& Uri, Banin. (2005). Formation of asymmetric one-sided metal-tipped semiconductor nanocrystal dots and rods. Nature Mater, 4, 855-863.

[9] Frenkel, A. (2007). Solving the 3D structure of metal nanoparticles. Zeitschrift Fur Kristallographie, 222, 605-611. 